\title{
Virtualization of Medication Delivery in Intensive Care Unit Layouts to Analyze Impact on Nurse Behavior
}

\author{
Smruti Keshani \\ Georgia Institute of Technology, USA \\ skeshani@gatech.edu \\ Matthew Swarts \\ Georgia Institute of Technology, USA \\ matthew.swarts@coa.gatech.edu
}

\begin{abstract}
This research design aims to understand medication delivery behavior under different spatial configurations through the virtualization of Intensive Care Unit (ICU) environments. Architectural visualization using virtual reality is used to demonstrate capabilities in capturing experiential data. Three different Intensive Care Unit floor plans were modeled and presented in 3D virtual environments. Tasks were developed to simulate nurses' activities during medication delivery to be analyzed for variables thought to affect nurse efficiency, nurse effectiveness, and satisfaction.
\end{abstract}

Keywords: 3D Virtual environment; Medication delivery; Space syntax; Hospital design.

\section{Introduction}

Studying the way in which nurses perform in an Intensive Care Unit, specifically under medication delivery tasks, has become one point of interest in research studies in the field of healthcare architecture. The way in which the physical environment (Shepley, 2003), social interactions (Penn, 1999), and cultural factors (Joseph, 2006) affect nurses productivity is of interest to researchers. By comparing what exists to what could be formulated based on chosen themes, simulations benefit research systems that use different problem solving techniques around one whole system, over a single-solution based problem (Eldabi, 2006). Though simulations have aided these studies immensely, the use of 3D virtual simulations may take these studies a step further. 3D virtual environments have the potential of creating a contribution to this type of spatial research by providing rapid evaluation to be run on various proposed designs. While time is conserved in the research process, virtual simulations also provide the opportunity for new innovations in design that before substantial time and money is invested in the construction, can be tested earlier in the design process to provide better decision making. The MacLeamy curve is the classical illustration demonstrating how more knowledge for decision making earlier in the design process can potentially lead to better outcomes (Curt, 2004). A clear study of how this technology can be integrated into existing research methods needs to be conducted and analyzed.

Exploration and investigation of existing spaces limits analysis only to existing structures in the present built environment which may be similar to a new design, but typically never identical. Security and privacy regulations in hospitals inhibit organizational, process, and space oriented research. The virtual approach provides the ability to manipulate unit layout that is not possible or practical in the real world setting. While occurrences of interactions and interruptions that might be encountered in a naturalistic observation of a real world environment are difficult to replicate, the isolation of a specific task (e.g. medication delivery) in a 3D virtual environment minimizes the need to perfectly mirror the real world.

\section{Background Review}

In our research design we look at the effects of a specific task in the virtual environment on nurse efficiency, effectiveness, and satisfaction, treating the built environment as an interface in which to perform the given tasks.

\section{Nurse effectiveness and efficiency}

One study has indicated a link between the communications involved in delivering quality care with the physical design of the given space (Dutta, 2008). The study found that in varying from a centralized unit to a decentralized unit, there was a dramatic reduction in interactions. Decentralized units were more preferred by participants, which suggest that a redesign of nursing stations towards decentralized units could be beneficial. Data from this study also showed a preference for a communication hub to be located at one place within the facility (Dutta, 2008). Further research is needed to clarify if more hubs would be beneficial to aid in the communication among nursing staff. 
Several case studies have been performed with space syntax analysis that have found a connection between building layouts and organizational behaviors such as movement patterns, physical presence, co-awareness, and unforced social interactions (Peponis, 2007; Choudary, 2009; Heo, 2009; Lu, 2009). These studies clarify how spatial measures of layout such as levels of visibility and accessibility are important in the study of nurses' movement, social communication, co-awareness and organizational performance (Cai, 2012). For example, Choudhary et. al. found in $\mathbf{2 0 0 9}$ that how many times a nurse will make it to a patient's room during his/her shifts can be predicted by the spatial properties of a nurse's given assignment. The model created from his study has an explanatory power that can predict the movement pattern of nurses in other hospital units of similar size and organizational processes (Choudhary, 2009).

The spatial design of a layout does more than just create opportunities for interaction and areas for information to propagate. Instead, workplace layout design creates a strategic framework that is constantly providing enrichment to the collective knowledge flowing through the organization. Spatial analysis can be used to produce a more quantitative description of a given layout in terms of spatial qualities. As a result of this quantitative description, one can see how the level of different interactions within a spatial context is altered by the design layout alone (Peponis, 2007). The amount of interactions could affect nurse effectiveness positively or negatively depending on the type of interaction. Additionally if the interaction were an interruption, this could also cause an increase in medication error, creating a negative effect of nurse effectiveness.

Another study argued that interactions within an office space, which can often be similar to a majority of the space allocated for nursing staff, did not have to be structured by the physical design of the environment. Instead, social organizational borders act the same as physical ones. Others have analyzed the way occupied spatial positions complement the traditional space syntax analysis methods. They did this by shifting from an analysis of movement to an analysis of interaction and created software for spatial modeling. The researchers concluded that rational choice theory and actor theory networks contribute important concepts and models to other researchers on how to perform proper spatial analysis of interactions (Markhede, 2010).

\section{Nurse emotional satisfaction}

While it is true that nurses gain self-satisfaction and feelings of happiness when helping to improve the lives of others, some thought must be given to the health of the nurses as well. The emotional stability and medical health of any employee can affect their performance greatly. Though external factors may contribute to this, internal interactions within one's workplace can have a larger impact. In 2002, Barrett investigated different levels of job satisfaction received as well as the burnout feeling and intention to leave work for the specialties of oncology and hematology nurses. The study concluded that $41.6 \%$ of the respondents were dissatisfied with the amount of time given to finish tasks for a given day. The opportunity for a higher rating of satisfaction may be possible by altering the spatial configuration of the healthcare facility to optimize for time. This would allow nurses to walk less and in return, finish their prescribed tasks in a shorter time. Further study on this topic can expand on how specific spatial factors affect the emotional states of nurses. The satisfaction of nurses might be predicted to improve as well if the space were configured to restrict or guide interruptions, which usually tend to create more stress on nurses, yet allow meaningful social interaction.

In another study performed by Seo, Choi, and Zimring in 2011, this issue of healthcare facility layout size was explored. Nurses were observed to see if a larger ICU unit with extra space for family members increased the walking distance between patient rooms as compared to a smaller ICU unit. This study showed, in contrast to the hypothesized belief that the larger unit did not increase the time nurses spent walking as compared to a smaller ICU unit. The nurses did however interact less and made fewer stops while walking in the various hallways. Evidence indicated that because fewer stops were made in-between patient room visits, the number of interactions a nurse had with other hospital personnel was decreased as well. Therefore, these nurses did not have the chance to accumulate additional task-irrelevant information from others, which could potentially contribute to mental strain by pushing themselves beyond their typical mental working capacity. This research study called for verification of the suggestion that co-visibility, substations, and the location in which medications are stored can reduce nurses' walking (Seo, 2011). In order to be able to understand these relationships better, researchers have given importance to developing ways to read these changes more efficiently. Instead of basing study models on general spatial relations, the act of narrowing these relations into categories allows for much clearer presentation of data to be read (Markhede, 2007).

\section{Research simulations}

Complex relationships and patterns can be constructed by modeling categories of organizational and spatial relations, which can then be used for simulation. While many types of simulation are used, discrete event simulations (DES) have often been used as decision making tools when one needs to "analyze processes, resources, and facility requirements to deliver [the] best clinical practice" (Gibson, 2007). Gibson used DES to look for ways to improve staff member's work environments as well as improve the quality and productivity of health services (Gibson, 2007). With an understanding of how to provide efficient flow through a healthcare facility, a study done at the Rush North Shore Medical Center Emergency Department Unit and Medical Telemetry Unit used a simulation to help evaluate two different units to reduce the variability within each. The simulation looked at the possibility of reducing a patient's length of stay and sought out ways to 
improve operations and communication between the two units. The study found that process improvement was able to increase if fewer patients were admitted or additional beds were added in the Medical Telemetry Unit (Blasak, 2003).

\section{Objectives}

Through the reconstruction of two previously researched Intensive Care Unit (ICU) wards into a 3D virtual environment, our study has two main goals: to analyze medication delivery behavior under different spatial configurations and to demonstrate the capabilities gained by using virtual reality over the physical built environment. Factors that come into consideration include: the amount of visibility into patients rooms each ICU unit offers nurses, the amount of time nurses spend walking for patient visits or medication delivery, and nurses' role in an ICU designed specifically for patient-centered care.

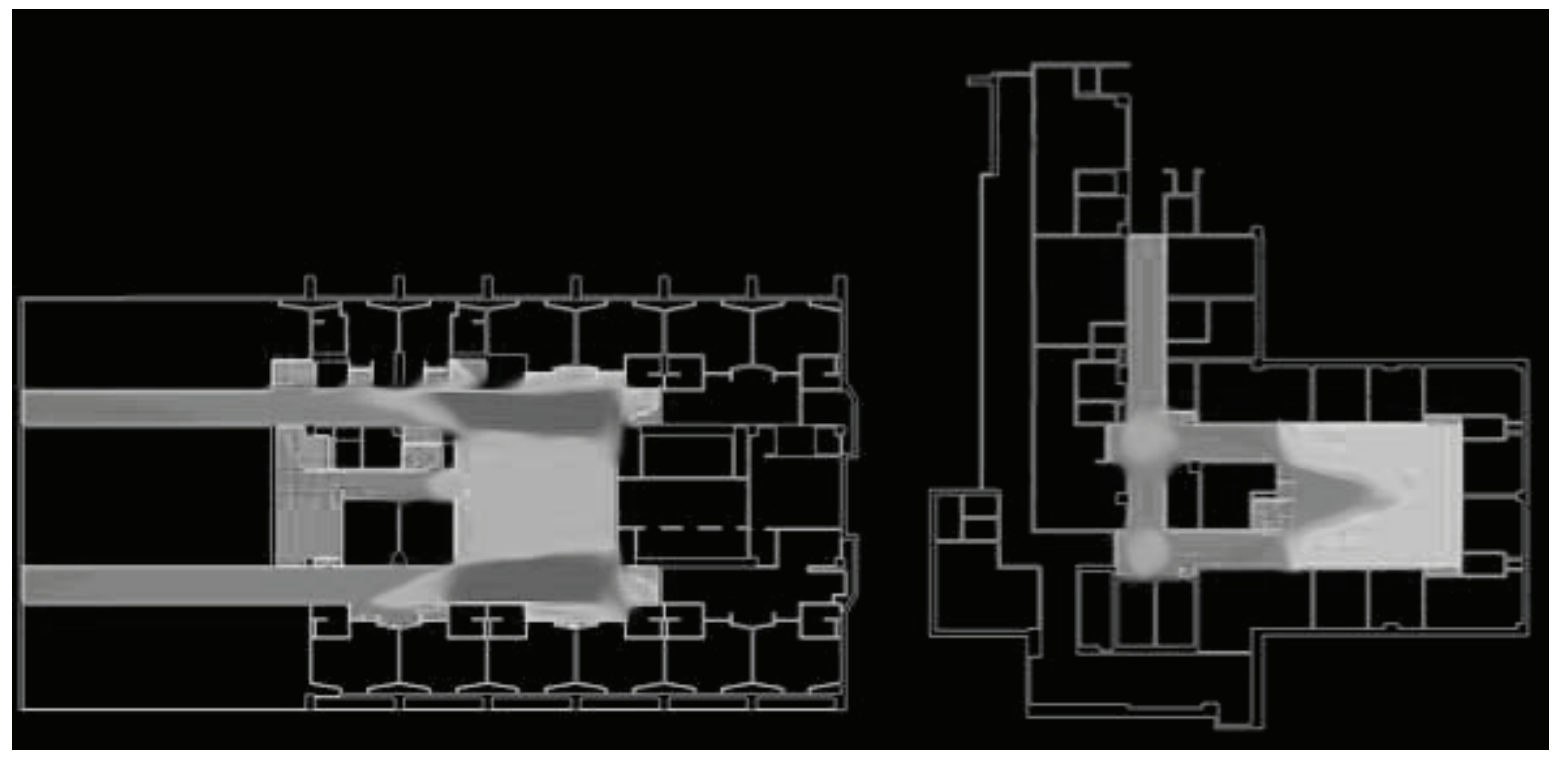

Figure 1: Mapping of overall visibility originated from DepthMap (Turner 2004).

The mapping of overall visibility, shown in figure 1 of each of the two existing ICU layouts, use red to indicate areas with high visibility and green to represent levels of low visibility. Our study intends to verify the suggestion, from the conclusion of Seo's study (2011), that visibility, substations, and the location in which medications are stored can reduce nurses' walking.

While the use of virtual reality to analyze behavior in generalized exterior environments has been exercised before, our research study is a further analysis of the conclusions of the study performed by Seo and a secondary validation study for the use of virtual simulations in healthcare architecture research. The primary dependent variables of this investigation are the effectiveness of correct medication delivery to patients, efficiency in delivery and task completion times, and the level of job satisfaction of nurses through survey.

This study aims to analyze medication delivery behavior under different spatial configurations, through the reconstruction of three ICU wards into a 3D virtual environment. Since only little research exists on the relationships among visibility, substations, and the location in which medications are stored can reduce nurses' walking (Seo 2011), it is important to conduct a study to determine whether these factors have any effects on medication delivery behaviors. In addition, since little research has been analyzed through the use of virtual reality for these purposes, we would like to validate its use for future studies. This required the identification of the variables that affect nurse performance, patient and family comfort, and the overall ICU atmosphere. Therefore, the specific aims of this project are to:

\section{Aim 1: Examine medication delivery behavior.}

Approach: Provide participants with a task list equipped with tasks similar to those of an actual ICU facility. Participants will have a range of tasks requiring different medication delivery functions (pill delivery, vaccine delivery, crushed pill delivery, IV installment).

Analysis: Observe and depict patterns in the method participants utilize. Particular attention will be focused on the number of trips taken to storage and patient rooms as well as walking routes chosen.

Impact: This will aid researchers in determining how to design ICU facilities that can facilitate in the medication delivery.

Aim 2: Determine the relationship between nurse behavior and medication delivery error rates.

Approach: Through comparing the amount of visibility each ICU unit offers to its nurses, the amount of time nurses spend walking for patient visits or medication deliveries, and nurses roles in an 
ICU designed specifically for patient-centered care. In addition, unforced interruptions nurses encounter in an ICU setting will be simulated.

Analysis: Nurses' responses to interruptions and interactions with other personnel in the environment. Their rate of medication delivery error and measure of satisfaction in response to these errors will be observed for a correlation.

Impact: More than aim 1, this understanding can aid designers in understanding how to reduce medication error rates, improve job satisfaction for nurses, increase patient care quality, and improve the effectiveness of ICU productivity.

Aim 3: Determine the possibility of $3 D$ virtual simulation environments to replace studies conducted in the limited physical built environment.

Approach: Run a 3D virtual simulation in the format as a secondary study of an existing study. Furthermore, expand upon the findings of the existing study.

Analysis: Compare the results of the virtual simulation to the original study for validation.

Impact: Introduce an opportunity to easily test more environments, including designs that have yet to be constructed. In addition, with this validation, the speed research is conducted, as well as the scope to which studies reach, may be extended greatly.

Overall, the goal of this project is to investigate how various hospital ICUs unit configurations impact the effectiveness, efficiency, and satisfaction of nurses as well as to investigate the possibility of validating the use of $3 \mathrm{D}$ virtual environments over the limited built environment in healthcare architecture research.

\section{Methodology}

This study contains four virtual environments. The first environment acts as the base environment. It allows users to become accustomed to the interaction methods and user interface. The remaining three environments are equipped with an exploration period to provide participants to gain a level of detail of the spatial layout, simulating a nurse's knowledge of the layout of his or her workplace. These three environments are presented in randomized order and allow for participants to complete tasks in the virtual ICU healthcare setting. This part simulates a day's worth of medication delivery for a few virtual patients in an 8-12 hour. While two of these three environments are constructed to prove the first and second aims of this project (see objective section), these environments are modeled directly after the ICUs in Seo study from 2011. By categorizing spatial types (Markhede 2007), a further categorization of medication supply locations can be examined. While one of the ICUs in Seo's study contains a centralized nursing station and the other has a decentralized nursing station, our virtual simulation of the units can demonstrate how the floor plan configuration affects performance in this way. In addition, based on the results from this primary study, the third virtual environment is designed to reduce medication errors and fix any problems or challenges that occur. Such changes may result from: medication supply locations, medication delivery types, nurse substations vs. centralized nurse stations, number of corners or turns in a layout, and length of hallways. These specific variables are varied in the last virtual environment. The basic model for these environments, mainly displaying the visual look of these environments is shown in figure 2.

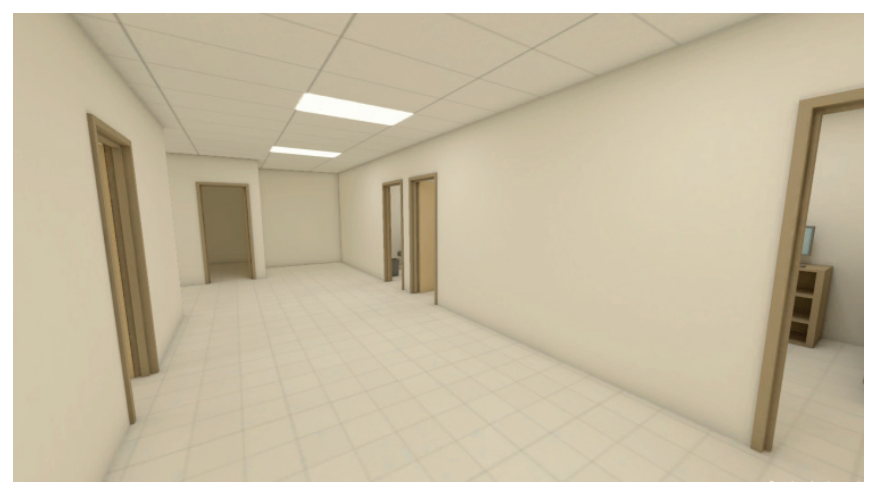

Figure 2: A basic model for each of the ICUs, crafted in AutoCAD and 3D Max Studio. The program will then be ran in Unity 3D.

Task lists for the virtual environments are given digitally to participants, allowing the ability to be checked off as completed. Our research team is concerned with the completion time of each user as well as the number of errors and distance walked. The virtual environments were modeled using AutoCAD, 3D Max Studio, and Revit. The simulation and protocols are developed in the Unity 3D game engine. In-depth analysis capabilities are made possible by running correlations between participant performance and space syntax measures.

\section{Conclusions}

Our virtual environment can provide empirical knowledge to designers earlier on how to arrange ICU units and also serve as a training tool for nurses recognizing the relationship among nurse stress, productivity, and medication errors. The ability to replay a session is useful for analyzing individual behavior. By collecting data through the virtual environment, we are able obtain more precise information on subject behavior which can be analyzed using traditional statistical measures. Even though a user in a virtual environment receives a different experience than in a real world hospital unit, the way in which one chooses to set up their medication cart or organize their day's work would be very similar. These decisions rely on a user's personal preference and habit. Therefore, we believe that though results may not be replicated exactly, the trends in which Seo (2011) was able to distinguish in his study will be observed in this virtual simulation. Namely, the presence of substations, and the location of medications stations can reduce nurse walking distance without much effect on the overall size of the unit. 
3D virtual environments have the potential of extending the methodologies available to spatial research by providing rapid empirical evaluation to be run on various proposed designs. Though human interaction inside virtual environments is different than in the physical environment, we can design experiments to minimize the differences by focusing on specific activities. Initial design knowledge can be created through these virtual simulations. In addition, these virtual simulations can provide input distributions to constructed simulations which could provide stakeholders with a sense of nurse's performance in different ICU layouts before too much time and money is invested. In particular, the factors of the built environment that affect effectiveness, efficiency, and satisfaction of a particular user group can be more efficiently explored to better understand how to produce best outcomes.

\section{Acknowledgments}

We would like to thank the Georgia Tech Presidential Undergraduate Research Award (PURA) program and to the SimTigrate Design Lab for their support in this research.

\section{References}

Penn, A., et al. (1999). "The space of innovation: interaction and communication in the work environment." Environment and Planning B: Planning and Design 26(2): 193-218.

Sepulveda, J. A., et al. (1999). The use of simulation for process improvement in a cancer treatment center. Proceedings of the 31st conference on Winter simulation: Simulation---a bridge to the future - Volume 2. Phoenix, Arizona, USA, ACM: 1541-1548.

Barrett, L. and P. Yates (2002). "Oncology/hematology nurses: a study of job satisfaction, burnout, and intention to leave the specialty." Australian Health Review 25(3): 109-121.

Tyson, G. A., et al. (2002). "The impact of ward design on the behavior, occupational satisfaction and well-being of psychiatric nurses." International Journal of Mental Health Nursing 11(2): 94.

Blasak, R. E., et al. (2003). Healthcare process analysis: the use of simulation to evaluate hospital operations between the emergency department and a medical telemetry unit. Proceedings of the 35th conference on Winter simulation: driving innovation. New Orleans, Louisiana, Winter Simulation Conference: 1887-1893.

Shepley, M. M. D., Kimberly (2003). "Nursing Unit Configuration and its Relationship to Noise and Nurse Walking Behavior: An AIDS/HIV Unit Case Study." The Academy Journal, AIA 6, October 2003.

Douglas, C. H. and M. R. Douglas (2004). "Patient-friendly hospital environments: exploring the patients' perspective." Health Expectations: An International Journal Of Public Participation In Health Care And Health Policy 7(1): 61-73.

Douglas, C. H. and M. R. Douglas (2005). "Patient-centered improvements in health-care built environments: perspectives and design indicators." Health Expectations: An International Journal Of Public Participation In Health Care And Health Policy 8(3): 264-276.

K. Preston White, J. (2005). A survey of data resources for simulating patient flows in healthcare delivery systems. Proceedings of the 37th conference on Winter simulation. Orlando, Florida, Winter Simulation Conference: 926-935.
Barach, P. D., Kenneth N. (2006). "Hospital Design Promoting Patient Safety." American Society for Healthcare Engineering International Conference, 2006.

Eldabi, T., et al. (2006). "Simulation modeling in healthcare: reviewing legacies and investigating futures." J Oper Res Soc 58(2): 262-270.

Joseph, A. (2006). "The Role of the Physical and Social Environment in Promoting Health, Safety, and Effectiveness in the Healthcare Workplace." The Center for Health Design (Paper \#3).

Straus, Eugene W., and Alex Straus. Medical Marvels: The 100 Greatest Advances in Medicine. Amherst: Prometheus Books, 2006.

Becker, F. (2007). "Nursing Unit Design and Communication Patterns; What is "Real" Work?" Health Environments Research and Design Journal 1(1): 58-62.

Gibson, I. W. (2007). An approach to hospital planning and design using discrete event simulation. Simulation Conference, 2007 Winter.

Markhede, H. K., Daniel (2007). "Positioning Analysis: social structures in configurative modeling." 6th International Space Syntax Symposium (Reference 069).

Peponis, J., et al. (2007). "Designing Space to Support Knowledge Work." Environment and Behavior 39(6): 815-840.

Burpee, Heather. "History of Healthcare Architecture." Mahlum. N.p., 2008. Web. 1 August 2013. www.mahlum.com.

Dutta, R. (2008 Jan). Influence of Nursing Unit Layout on Staff Communication and Interaction Patterns. These presented to Cornell Univ Grad School.

Choudhary, R., et al. (2009). "A predictive model for computing the influence of space layouts on nurses' movement in hospital units." Journal of Building Performance Simulation 3(3): 171-184.

Henrich, A. C., Marilyn P; Bafna, Sonit; Choudhary, Ruchi; Heo, Yeonsook; Skeierczynski, Boguslaw A (2009). "Unit-Related Factors That Affect Nursing Time with Patients- Spatial Analysis of the Time and Motion Study." HERD.

Heo, Y. C., Ruchi; Bafna, Sonit; Hendrich, Ann; Chow, Marylyn P. (2009). "A Modeling Approach for Estimating the Impact of Spatial Configuration on nurses' Movement." 7th International Space Syntax Symposium: Ref 041.

Lu, Y. P., John; Zimring, Craig (2009). "Targeted Visibility Analysis in Buildings: Correlating Targeted Visibility Analysis with Distribution of People and Their Interactions within an Intensive Care Unit." 7th International Space Syntax Symposium.

Markhede, H. (2010). "Spatial positioning: method development for spatial analysis of interaction in buildings." Trita-ARK. Forskningspublikationer: iii, 23.

Cai, H. Z., Craig (2012). "Out of Sight, Out of Reach: Correlating spatial metrics of nurse station typology with nurses' communication and Co-Awareness in an intensive care unit." 8th International Space Syntax Symposium.

Seo, Hyun Bo, Choi, Y., \& Zimring, C. (2011). Impact of Hospital Unit Design for Patient-Centered Care on Nurses' Behavior. Environment and Behavior, 43(4), 443-468.

Turner, A. (2004). "Depthmap 4 | A Researcher's Handbook", Bartlet School of Graduate Studies, UCL, London. http://www.vr.ucl.ac.uk/depthmap/handbook/depthmap 4r1.pdf 